\title{
Nonequivalence of updating rules in evolutionary games under high mutation rates
}

\author{
G. A. Kaiping, ${ }^{1, *}$ G. S. Jacobs, ${ }^{2}$ S. J. Cox, ${ }^{1}$ and T. J. Sluckin ${ }^{2}$ \\ ${ }^{1}$ Computational Engineering and Design, University of Southampton, Southampton, United Kingdom \\ ${ }^{2}$ Applied Mathematics, University of Southampton, Southampton, United Kingdom
}

(Received 10 April 2014; published 30 October 2014)

\begin{abstract}
Moran processes are often used to model selection in evolutionary simulations. The updating rule in Moran processes is a birth-death process, i. e., selection according to fitness of an individual to give birth, followed by the death of a random individual. For well-mixed populations with only two strategies this updating rule is known to be equivalent to selecting unfit individuals for death and then selecting randomly for procreation (biased death-birth process). It is, however, known that this equivalence does not hold when considering structured populations. Here we study whether changing the updating rule can also have an effect in well-mixed populations in the presence of more than two strategies and high mutation rates. We find, using three models from different areas of evolutionary simulation, that the choice of updating rule can change model results. We show, e. g., that going from the birth-death process to the death-birth process can change a public goods game with punishment from containing mostly defectors to having a majority of cooperative strategies. From the examples given we derive guidelines indicating when the choice of the updating rule can be expected to have an impact on the results of the model.
\end{abstract}

DOI: 10.1103/PhysRevE.90.042726

PACS number(s): 87.23. $-\mathrm{n}$

\section{INTRODUCTION}

Evolutionary models are used to explore a diverse range of research topics from cancer cell spread [1] and particle systems [2] to the evolution of language traits [3] and cooperation [4]. As such, results from these models greatly inform our characterization of many systems, with implications for how we view the world and our own social behavior. Predictions and policies are based on findings from evolutionary game theory [5,6]. Given this, an awareness of how implicit assumptions in evolutionary models can impact their outcomes is vital.

The underlying framework of any evolutionary model must incorporate an updating rule. This describes the core evolutionary properties of the system to be explored. In this work, we consider how different updating rule formulations can lead to varied model results, while appearing to capture similar evolutionary properties. Our focus is on a subtlety of the implementation of selection - whether fitness advantage is rewarded by increased survival or increased reproduction.

There is a small number of updating rules regularly employed in models with fixed population size. We focus on three of these (birth process, death process, and link dynamics) as described below. We postpone their formal definition and detailed description to Sec. II.

Other updating rules have also been used to model evolutionary processes, but much more rarely. The three updating rules compared in this article are based on replication (or imitation) of one individual in each time step. It is known that other families of updating rules, such as myopic best response, can lead to entirely different evolutionary dynamics $[7,8]$.

Moran [9] introduced a class of evolutionary models which employ an updating rule known as the birth-death process (BP) [10] or (biased) invasion process [11]. In these models, the fitness of an individual gives the probability that this individual

*Corresponding author: gereon.kaiping@ soton.ac.uk produces offspring in a given time step. This type of model is intermediate between the discrete-time Wright-Fisher model [12], in which one time step corresponds to one generation of the finite population, and continuous-time approaches using differential equations and infinite populations. The Moran process permits the study of overlapping generations in a finite population of fixed size, using a discrete-time Markov chain. The transition probabilities associated with the birth-death process have comparatively easy algebraic expressions (see, e. g., Ref. [3]) and are therefore often used where algebraic expressions of derived properties like fixation probabilities are relevant. The BP updating rule appears far more frequently in evolutionary models literature than the following two.

The death-birth process (DP) [10] was introduced by Williams and Bjerknes $[13,14]$ to study tumor growth and has been independently studied in the context of particle systems under the name "voter model" $[1,2,15,16]$. We note that although this updating rule is called a model in the context of particle systems, we will reserve the term "model" in this article for a combination of updating rule, population structure, and underlying game. By contrast with BP, in which fitness is proportional to the stochastic birth rate of an individual, in the death-birth process fitness rather governs an individual's chance of survival.

The link dynamics updating rule (LD) [17] arose from the study of structured populations and focuses on pairwise interactions. In most cases, a population structure is given in terms of a (static) graph [18], in which nodes correspond to individuals in the population. A link between two nodes then indicates that in the updating step of the model, one of these nodes can replace the other one. The effect of different population structures on the evolutionary process has been studied for several years [19-23]. This paper, on the other hand, considers only unstructured, well-mixed populations. A variant of LD was introduced by Szabo and Tőke [24] and has been studied under the names pairwise comparison evolutionary process [25] and Fermi process [26] for the prisoner's dilemma on lattices. 
Of particular interest in evolutionary models is the process of fixation of a single mutant in a population of residents. Relevant quantities obtained from a given model are the fixation probability, i. e., the probability that a single mutant allele overtakes a homogenous population of a different allele and consequent fixation times.

In many evolutionary models, it is a reasonable assumption that at any given point in time, only two different alleles are present in the population. Given a sufficiently small mutation rate $\mu$, the probability that a mutant enters a population already possessing more than one type of allele in it may be regarded as negligible [27,28]. Thus the population will always only contain residents and possibly at most one type of mutant.

If the population structure is homogenous, populations where this assumption is true show equal fixation probabilities for all three of the evolutionary processes introduced above, as has been shown by Antal et al. [29]. In generic graphs, on the other hand, this equivalence among BP, DP, and LD is lost [29]. This result entailed investigations into how different updating rules lead to different evolutionary dynamics on various graph structures [30,31].

In this article we investigate the differences between the different updating rules mentioned above when instead dismissing the two-allele assumption. The error induced by that assumption has been previously studied by Wu et al. [26]. Their study employs the Fermi process as updating rule on a well-mixed model of population size $N$. Of particular interest is their result for coexistence games, in which the best reply to every strategy is a different strategy. They find that the two-allele approximation is reliable only if the mutation rate $\mu$ behaves as $O\left(N^{-\frac{1}{2}} \exp (-N)\right.$ ) (where $N$ is the population size). This is a very restrictive requirement, which is unlikely to hold in practice even for small populations. The expected time between mutations needs to be significantly longer than the age of the universe in order for mutation to be a negligible factor.

For example, using Eqs. (3) and (4) below, we see that the expected consensus and fixation times of the anticoordination game with payoff matrix

$$
\left(\begin{array}{ll}
0 & 1 \\
1 & 0
\end{array}\right),
$$

among 100 players, for any of the three updating rules, is about $1.3 \times 10^{30}$ time steps, which at a generation time as low as 10 min corresponds to $1.8 \times 10^{13}$ times the age of the universe. In this paper we therefore drop the assumption that mutation rates are so low that the fixation time is negligible with respect to the time between mutations. Depending on the circumstances, the implications of this relaxation may differ. For some cases, it will be necessary to explicitly study high mutation rates in a model. Alternatively, the effects of high mutation can be studied by allowing more than two strategies to be present at one time step in a given system.

The organization of this paper is as follows. In Sec. II, we will recall some general observations from evolutionary game theory before describing the update rules, BP, DP, and LD in more detail, giving example calculations from basic evolutionary game theory for each.

We will then present three models that show differences in behavior for the three updating rules in Sec. III. Model 1 (III B) is a model from evolutionary linguistics, which we use to examine the effect of different updating rules in the context of high mutation rates. We will show that for high mutation values, changing the updating rule affects not only the quantitative values of the equilibria of a model but also the topology of the equilibrium space.

For the other models, we look at cases where more than two strategies are present in the system. Model 2 (III C) is a simple model from abstract game theory. We show explicitly that the equilibria of the model are identical for the three updating rules. Interestingly, however, the choice of updating rule does impact both the quantitative geometry of the equilibria and their basins of attraction.

We continue using the example of the optional public goods game with peer punishment (model 3, IIID). We use this example to illustrate that the choice of updating rule has a significant quantitative influence also in well-known models from the study of the evolution of cooperation.

Finally, in Sec. IV, we conclude with a general discussion of the properties of the updating rules studied. We suggest heuristic criteria as to when changing the updating rule significantly alters the results of the model and in what contexts to employ which updating rule.

\section{UPDATING RULES}

Updating rules such as BP, DP, or LD define a discrete Markov process. The states correspond to all possible configurations of the underlying graph. In the commonly treated case of well-mixed populations, for example, every state is completely described by giving the number of individuals having a particular allele. The transition probabilities between states are derived from the fitness values of the alleles, according to the updating rule. The rules considered here are defined such that the population size will be constant.

In order to provide context for our more general study, we first recall well-known results for Markov processes in well-mixed two-allele populations. In what follows we employ the notation and conventions of Antal and Scheuring [32].

Consider a well-mixed population of players, each following one of two different strategies $\mathrm{A}$ and $\mathrm{B}$. The state of a population of size $N$ is defined by a single integer $0 \leqslant n \leqslant N$, giving the number of individuals in the population following strategy A. Suppose also that at each time step each individual in the population plays a random other individual in a symmetric game. Let the payoff for the row player be given by the payoff matrix

\begin{tabular}{l|ll} 
& $\mathrm{A}$ & $\mathrm{B}$ \\
\hline $\mathrm{A}$ & $a$ & $b$ \\
$\mathrm{~B}$ & $c$ & $d$
\end{tabular}

for $a, b, c, d>0$.

The fitness of an individual playing strategy A or B, respectively, is defined to be its expected payoff, that is,

$f_{A}(n)=\frac{a(n-1)+b(N-n)}{N-1}, \quad f_{B}(n)=\frac{c n+d(N-n-1)}{N-1}$.

The transition probabilities

$$
\begin{aligned}
\lambda_{n} & =P(n \rightarrow n+1) \\
& =P(\text { one of type A procreates }) P(\text { one of type B dies })
\end{aligned}
$$




$$
\begin{aligned}
\mu_{n} & =P(n \rightarrow n-1) \\
& =P(\text { one of type } \mathrm{B} \text { procreates }) P(\text { one of type A dies })
\end{aligned}
$$

are derived from $f_{A}(n)$ and $f_{B}(n)$. An updating rule defines how to obtain $\lambda_{n}$ and $\mu_{n}$ from $f_{A}(n)$ and $f_{B}(n)$. We discuss this in more detail below. Calculations for Moran's BP are given by Eqs. (5) and (6). The BP updating rule is one of many consistent routes from $f_{A}(n), f_{B}(n)$ to $\lambda_{n}, \mu_{n}$. However, whatever route is chosen, an invariant property of $\left(\lambda_{n}, \mu_{n}\right)$ is that

$$
\begin{aligned}
\frac{f_{B}(n)}{f_{A}(n)}= & \frac{\mu_{n}}{\lambda_{n}}=\frac{P(n \rightarrow n-1)}{P(n \rightarrow n+1)} \\
= & \frac{P(\text { one of type B procreates })}{P(\text { one of type B dies })} \\
& \times\left[\frac{P(\text { one of type A procreates })}{P \text { (one of type A dies })}\right]^{-1} .
\end{aligned}
$$

Thus $\mu / \lambda$ is the quotient of the stochastic growth rates of the A and B populations, justifying the use of the term fitness in this context, even when the updating rule is not BP.

For any two-allele Markov model with one individual dying and one individual being born every step, define

$$
q_{n}=\prod_{j=1}^{n} \frac{\mu_{j}}{\lambda_{j}} \quad q_{0}=1 \quad s_{n, m}=\sum_{k=n}^{m} q_{k} .
$$

We remark that $q_{n}$ and $s_{n, m}$ are polynomials in the variables $\left\{x_{n}=\frac{\mu_{n}}{\lambda_{n}} \mid n=1 \ldots N\right\}$, with no single $\mu$ or $\lambda$ appearing in the coefficients. Furthermore, define the probability $\epsilon_{n}$ that the system will be absorbed by state 0 . In terms of $s_{n, m}$ this can be expressed as

$$
\epsilon_{n}=\frac{s_{n, N-1}}{s_{0, N-1}}
$$

We recall that state 0 corresponds to a state in which all individuals have allele $\mathrm{A}$. The other absorbing state of each individual playing $\mathrm{B}$ is denoted by $N$. The fixation probability of a single mutant of strategy $\mathrm{A}$ in a population of $N-1$ individuals following strategy $\mathrm{B}$ is then given by

$$
\rho_{A B}=\left(1-\epsilon_{1}\right)=\frac{1}{s_{0, N-1}} .
$$

The expected time until fixation of a single mutant, given that the mutant will fixate, is the mean conditional exit time,

$$
t_{\mathrm{fix}}=\sum_{n=1}^{N-1} \frac{s_{0, n-1} s_{n, N-1}}{\lambda_{n} q_{n} s_{0, N-1}} .
$$

In the models we explore below, there are three or more possible strategies. A quantity of relevance in the three-allele problem is the expected time $t_{\text {cons }}$ until a two-allele system fixates in either of the two absorbing states. If the mutation rate $\mu$ is of the order of magnitude of $t_{\text {cons }}{ }^{-1}$ or higher, the probability of of a mutation occuring in a nonfixated system can no longer be considered negligible. The consensus or unconditional mean exit time $t_{\text {cons }}$ is defined [33] as the expected time until the system reaches either of the absorbing states when starting at $n=1$,

$$
t_{\text {cons }}=\sum_{n=1}^{N-1} \frac{s_{n, N-1}}{\lambda_{n} q_{n} s_{0, N-1}} .
$$

By definition, $\frac{\mu_{j}}{\lambda_{j}}$ has the same value for all three updating rules. From Eqs. (1) and (2) it follows that in the general case the fixation probabilities $\rho_{A B}$ will be independent of the specific updating rule chosen. By contrast, the additional $\lambda_{n}$ terms in Eqs. (3) and (4) can mean significant deviations between the expected unconditional mean fixation times when comparing the three updating rules.

In what follows we discuss some specific details of the BP, DP, and LD processes.

\section{A. Birth-death process}

The Moran process, introduced by P. A. P. Moran in 1958 [9], is frequently used as an updating rule in evolutionary simulations. It is also known as the BP or biased birth-death process. In every time step, one random individual is replaced by a copy of an individual randomly chosen with probability proportional to its fitness. An individual can be replaced by a copy of itself.

The transition probabilities in the two-allele case are therefore given by

$$
\begin{gathered}
P(n \rightarrow n-1)=\mu_{n}=\frac{(N-n) f_{B}(n)}{n f_{A}(n)+(N-n) f_{B}(n)} \frac{n}{N}, \\
P(n \rightarrow n+1)=\lambda_{n}=\frac{n f_{A}(n)}{n f_{A}(n)+(N-n) f_{B}(n)} \frac{N-n}{N}, \\
P(n \rightarrow n)=1-\mu_{n}-\lambda_{n}, \\
P(n \rightarrow j)=0 \text { otherwise, }
\end{gathered}
$$

so we have $\frac{\mu_{n}}{\lambda_{n}}=\frac{f_{B}(n)}{f_{A}(n)}$ and therefore

$$
\begin{aligned}
\rho_{A B} & =\frac{1}{\sum_{k=0}^{N-1} \prod_{l=1}^{k} \frac{f_{B}(l)}{f_{A}(l)}}, \\
& =\frac{1}{\sum_{k=0}^{N-1} \prod_{l=1}^{k} \frac{c l+d(N-l-1)}{a(l-1)+b(N-l)}} .
\end{aligned}
$$

For the neutral case $a=b=c=d$ this gives us a fixation probability $\rho_{A B}=\frac{1}{N}$. For the case of a mutant with a constant fitness advantage $a=b=r>1=c=d$ we obtain the wellknown result [9] that the fixation probability is given by $\rho_{A B}=$ $\frac{1}{\sum_{k=0}^{N-1} r^{-k}}=\frac{1-r^{-1}}{1-r^{-N}}$.

By way of example, we can calculate the expected times until fixation for the prisoner's dilemma with payoff matrix

$$
Q=\left(\begin{array}{ll}
1 & 5 \\
0.1 & 3
\end{array}\right)
$$

for a population size of 100. From Eq. (3) we obtain that the conditional fixation time $t_{\mathrm{fix}} \approx 1264.5$ for both a single cooperator among defectors and a single defector among cooperators. Following Eq. (4), the average time until the system is in any fixated state again after starting from a single defector is introduced into a population of cooperators is approximately 569.6 updating steps, while for a single 
cooperator among a population of defectors is close to the life expectancy of that cooperator, because the probability of him or her reproducing is near 0 . Therefore in this case we have $t_{\text {cons }} \approx 105.3$.

\section{B. Death-birth process}

In the ecological and evolutionary literature, fitness usually corresponds to the mean number of adult offspring per generation. High fitness can thus imply a high fecundity, as seen in the BP. Alternatively, high fitness can be expressed as high probability of survival. This is modelled by the DP updating rule. Just as in BP, in every time step an individual chosen randomly according so some distribution is replaced by a copy of a random individual. However, in DP, the fecundities or birth probabilities of all individuals are assumed to be equal, while the death probabilities are taken to be proportional to the reciprocal of the fitness.

The transition probabilities for this updating rule are thus given by

$$
\begin{aligned}
P(n \rightarrow n-1) & =\mu_{n}=\frac{N-n}{N} \frac{n / f_{A}(n)}{n / f_{A}(n)+(N-n) / f_{B}(n)} \\
& =\frac{N-n}{N} \frac{n f_{B}(n)}{n f_{B}(n)+(N-n) f_{A}(n)}, \\
P(n \rightarrow n+1) & =\lambda_{n}=\frac{n}{N} \frac{(N-n) f_{A}(n)}{n f_{B}(n)+(N-n) f_{A}(n)}, \\
P(n \rightarrow n) & =1-\mu_{n}-\lambda_{n}, \\
P(n \rightarrow j) & =0 \text { otherwise. }
\end{aligned}
$$

Note that $\frac{\mu_{n}}{\lambda_{n}}=\frac{f_{B}(n)}{f_{A}(n)}$, just as for the birth-death process, and therefore the fixation probability for the DP is indeed the same as for the BP [29].

This is not, however, true for the fixation times in the prisoner's dilemma example with payoff matrix $Q$ as in Eq. (7). Here we have a slightly lower conditional fixation time of $t_{\text {fix }} \approx 1191.0$ for both cases. The expected time until the system is in any fixated state, after starting from a single defector, is slightly longer than in the BP, with a value of 575.3 time steps. Although a single cooperator in a population of defectors has a relatively large probability of reproduction compared to the $\mathrm{BP}$, at $\frac{1}{100}$, the likelihood of dying is very high. As a result, the expected time to elimination is $t_{\text {cons }}=11.4$, significantly less than in the BP case.

\section{Link dynamics}

The third frequently applied updating rule that we study, LD, proceeds as follows. At every time step a random link of the graph describing the population structure is chosen. Two individuals are adjacent to the edge. One of these is chosen randomly as donor $k$ with probability proportional to its fitness. The other individual $j$ is then replaced by a copy of $k$.

In many cases of numerical simulation, the link dynamics updating rule has a significant computational benefit over BP and DP. The LD rule requires the calculation of the fitness values for only two individuals in every time step. BP and DP, by contrast, necessarily rely on knowing the fitness values of every individual in the population.
For the well-mixed case, the population structure contains an edge from every individual to every individual, including itself. The population structure is thus given by a fully connected graph with self-loops. This is consistent with the assumption made in the previous two subsections for BP and DP, namely that in the same time step an individual can both procreate and die, thereby replacing itself.

The probability that a self-loop is randomly selected as focal link is $\frac{N}{\frac{N(N-1)}{2}+N}=\frac{2}{N+1}$. In the absence of mutation, this implies that nothing happens for such a time step. A model with self-loops corresponds to a model without self-loops in which there is a probability of $\frac{2}{N+1}$ for every time step that nothing happens in that step. We can thus transform one approach to the other with a linear rescaling of time by a factor $\frac{N-1}{N+1}$, such that results given for one approach are easily translated to the alternative.

A related well-known updating rule for well-mixed populations is the Fermi process [33]. In the same way as in the LD updating rule, a random link in the population is chosen. However, in case of the Fermi process, both individuals are equally likely to be chosen as donor $k$. In contrast with LD, where $j$ is always replaced by a copy of $k$, for the Fermi process this replacement happens only with a probability of $\frac{1}{1+\exp \left(\beta\left[\pi_{j}-\pi_{k}\right]\right)}$.

This probability distribution is known as the Fermi distribution and is eponymous for the updating rule. Here $\beta>0$ is a constant describing the strength of selection. In the context of the Fermi distribution, it can be interpreted as an inverse temperature. Lower $\beta$ corresponds to higher randomness of the system and thus weaker selection.

The quantities $\pi_{x}$ are payoff functions [26]. In contrast to the fitness functions $f_{x}$ employed in the other updating rules considered here, these payoff functions may take negative values. We also note that, while for BP, DP, and LD every time step corresponds to precisely one individual procreating and one individual dying, in the Fermi process there is a probability of $1-\frac{1}{1+\exp \left(\beta\left[\pi_{j}-\pi_{k}\right]\right)}$ in every time step that no individual procreates and none die.

In fact, we see that the Fermi process is a variant of LD if we define a fitness function $f_{x}=\exp \left(\beta \pi_{x}\right)$. In the LD case, the transition probabilities are

$$
\begin{aligned}
P(n \rightarrow n-1) & =\mu_{n}=2 \frac{n}{N} \frac{N-n}{N} \frac{f_{B}(n)}{f_{A}(n)+f_{B}(n)}, \\
P(n \rightarrow n+1) & =\lambda_{n}=2 \frac{N-n}{N} \frac{n}{N} \frac{f_{A}(n)}{f_{A}(n)+f_{B}(n)}, \\
P(n \rightarrow n) & =1-\mu_{n}-\lambda_{n}, \\
P(n \rightarrow j) & =0 \text { otherwise. }
\end{aligned}
$$

For the Fermi process, we have

$$
\begin{gathered}
P^{F}(n \rightarrow n-1)=\mu_{n}^{F}=\frac{n}{N} \frac{N-n}{N} \frac{f_{B}(n)}{f_{A}(n)+f_{B}(n)}=\frac{1}{2} \mu_{n}, \\
P^{F}(n \rightarrow n+1)=\lambda_{n}^{F}=\frac{N-n}{N} \frac{n}{N} \frac{f_{A}(n)}{f_{A}(n)+f_{B}(n)}=\frac{1}{2} \lambda_{n}, \\
P^{F}(n \rightarrow n)=1-\mu_{n}^{F}-\lambda_{n}^{F}=\frac{1}{2}\left(1-\mu_{n}-\lambda_{n}\right)+\frac{1}{2}, \\
P^{F}(n \rightarrow j)=0 \text { otherwise. }
\end{gathered}
$$


Comparing Eqs. (10) and (11) indicates a probability of $\frac{1}{2}$ in each time step that the Fermi process behaves like LD and a probability of $\frac{1}{2}$ that it remains in the same state as before. As a result, one can translate times and expected times obtained through LD to the Fermi process by rescaling, stochastically or exactly respectively, by a factor of 2 .

From the terms for $\mu_{n}$ and $\lambda_{n}$ in Eqs. (10) and (11) we see that, as for BP and DP, we have $\frac{\mu_{n}}{\lambda_{n}}=\frac{f_{B}(n)}{f_{A}(n)}$. Hence the same fixation probabilities for LD and Fermi process are the same as for those two updating rules.

When comparing the transition probabilities of the three updating rules, recalling Eqs. (5), (9), and (10), we see that

$$
\begin{aligned}
\mu_{n}^{\mathrm{BP}} & =\frac{n}{N} \frac{(N-n) f_{B}(n)}{n f_{A}(n)+(N-n) f_{B}(n)}, \\
\mu_{n}^{\mathrm{DP}} & =\frac{N-n}{N} \frac{n f_{B}(n)}{n f_{B}(n)+(N-n) f_{A}(n)}, \\
\mu_{n}^{\mathrm{LD}} & =2 \frac{n}{N} \frac{N-n}{N} \frac{f_{B}(n)}{f_{A}(n)+f_{B}(n)}, \\
& =\frac{2}{\frac{1}{\mu_{n}^{\mathrm{BP}}}+\frac{1}{\mu_{n}^{\mathrm{DP}}}} .
\end{aligned}
$$

Thus $\mu_{n}^{\mathrm{LD}}$ is the harmonic mean of the analogous quantities $\mu_{n}^{\mathrm{BP}}$ and $\mu_{n}^{\mathrm{DP}}$. The transition probability $\lambda_{n}^{\mathrm{LD}}$ is likewise the harmonic mean of the corresponding quantities for BP and DP. The LD updating rule is therefore expected to lead to behavior that is intermediate between these two rules.

By way of example we see that for the fixation times in the prisoners' dilemma with payoff matrix $Q$ from Eq. (7), we get precisely the arithmetic mean of the corresponding values from the birth-death process and death-birth process. We obtain $t_{\text {fix }} \approx 1227.7$ for both a single cooperator among defectors and a single defector among cooperators. The expected time until the system is in any fixated state, after starting from a single defector, is 572.5 time steps, and for starting from a single cooperator in a population of defectors we have $t_{\text {cons }} \approx 58.4$.

Due to the exponential function deriving fitness from payoff, and the additional parameter $\beta$, a direct comparison between the Fermi process and the BP, DP, and LD updating rule is outside the scope of this paper. For a detailed analysis of the fixation times for the Fermi process for $\beta \ll 1$ we refer to Altrock and Traulsen [33]. In this reference the authors also give a comparison between the birth-death process generalized to contain a selection strength parameter and the Fermi process for low selection strength.

It is the equality of the fixation probabilities of BP, DP, and LD which motivates Antal et al. [29] to refer to these three updating rules as equivalent for homogenous graphs. We remark that this is a very strong term for this specific property. We have indeed seen that for the simple cases of well-mixed mutation-free two-strategy games considered in this section the long-term behavior of the model, as expressed by the fixation probability, does not change under different updating rules. The vast difference in the consensus times, however, serves as a first indication that applying different updating rules may influence model results. In the next section we will show that different updating rules can indeed lead to different long-term behavior of otherwise equal evolutionary models.

\section{EXAMPLE MODELS}

We will now present three examples of systems in which a change to the updating rule also significantly changes its dynamics. Just as for the results given in the previous section, all three examples feature well-mixed populations of discrete strategies. For each of the three examples, we will first describe the model in general before giving the results for each of BP, DP, and LD and comparing the results. However, in contrast with the previous section, the examples studied here will not assume that the mutation rate is small enough to be negligible for the dynamics of a nonfixated system. Furthermore, fitness functions will not be restricted to the expected payoff from two-player games but can be arbitrary nonzero functions.

\section{A. Methodology}

Mean-field theories are mathematically simplified methods for analyzing stochastic well-mixed systems. Using this approach, the Markov chains of an evolutionary model with a large population can be approximated by systems of ordinary differential equations. We will use both the mean-field approximation as well as stochastic methods to examine the example models below.

On the one hand, the large-population approximation of the mean-field theory has the effect of smoothing the stochastic effects in a system, which are of particular interest when studying frequent mutation. On the other hand, the mean-field theory is often more computationally efficient than numerical simulations or the calculation of a Markov chain stationary distribution.

Furthermore, the system of ordinary differential equations allows us to study the equilibria of the mean-field theory of an evolutionary system. Equilibria are characterized by the fact that the differentials of the unknown functions are zero for those states. Depending on their stability, these equilibria can be repellers, saddle points, or attractors. If a state is an attractor, a small disturbance will lead to the system returning to that same state. Attractors of the system are therefore evolutionarily stable states. Saddles and repellers lie on the boundaries between the basins of attraction of different evolutionarily stable states. Thus, mean-field theory is useful for cataloguing all evolutionarily stable states, together with their basins of attraction.

To obtain the system of analytical ordinary differential equations corresponding to each updating rule, we generalize the method described by Traulsen et al. [34] to the higher-dimensional case. Let $P^{\tau}(\xi)=P^{\tau}\left(\xi_{1}, \xi_{2}, \ldots, \xi_{n}\right)$ be the probability that a process with $n$ alleles is in state $\xi$ at time $\tau$. Let $F^{i}(\xi)$ be the fecundity, i. e., the probability that an individual of type $i$ is born when the system is in state $\xi$, and $M^{i}(\xi)$ the mortality rate of type $i$. Define $e_{i}$ to be the $i$ th unit vector $(0, \ldots, 0,1,0, \ldots, 0)$. Then the stochastic process is given by the master equation

$$
\begin{aligned}
P^{\tau+1}(\xi)-P^{\tau}(\xi)= & \sum_{j=1}^{n} \sum_{i=1 ; i \neq j}^{n}\left[P^{\tau}\left(\xi-e_{i}+e_{j}\right)\right. \\
& \times F^{i}\left(\xi-e_{i}+e_{j}\right) M^{j}\left(\xi-e_{i}+e_{j}\right) \\
& \left.-P^{\tau}(\xi) F^{i}(\xi) M^{j}(\xi)\right] .
\end{aligned}
$$


Let $N=\sum_{i=1}^{n} \xi_{i}$ be the population size. Introducing $x=$ $\xi / N, t=\tau / N$, and $\rho(x, t)=N P^{N t}(N x), \rho$ is given by a Taylor series in $x$ and $t$. Ignoring higher-order terms in $N^{-1}$, this series yields the partial differential equation

$$
\frac{d}{d t} \rho(x, t)=-\frac{d}{d x}[a(x) \rho(x, t)]+\frac{1}{2} \frac{d^{2}}{d x^{2}}\left[b^{2}(x) \rho(x, t)\right]
$$

with $a(x)=F(x)-M(x)$ and $b(x)=\sqrt{N^{-1}[F(x)+M(x)]}$. For $N \rightarrow \infty$ the diffusion term $b \rightarrow 0$, and we get a system of ordinary differential equations,

$$
\dot{x}=F(x)-M(x) .
$$

This means that in the infinite population limit, the change $\dot{x}_{i}$ of the relative frequency of allele $i$ is given by the difference of its probabilistic fecundity $F_{i}(x)$ and its probabilistic mortality $M_{i}(x)$.

Model equilibria therefore correspond to states in which $M(x)=F(x)$. In systems without mutation this is equivalent to the condition that the fitnesses of all strategies present be equal. This condition is independent of the updating rule. In the presence of mutation it might by analogy be reasonable to assume that a change of updating rule might do no more than perturb the precise value of an equilibrium, while leaving the topology of the equilibrium structure intact. This is, however, not the case, as the first example (III B) will show.

By contrast, for stochastic systems with finite populations the notion of equilibrium is less clear. If the mutation probability of the model is greater than zero, the underlying Markov chain is irreducible. Thus the chain has a unique stationary probability distribution $\pi$ [35]. As a real-valued function on a discrete space, this distribution has local maxima, minima, and saddle points. These special points, however, do not correspond to equilibria of the mean-field theory, and the notion of a basin of attraction is hardly applicable. In particular, the stationary distribution does not contain any information on basins of attraction, because, heuristically, basins of attraction correspond to short-term behavior, while the stationary distribution is by definition concerned with only the long-term limit. When analyzing our model 3 III D in terms of its long-term behavior, we will therefore resort to summary statistics.

\section{B. Model 1: Evolutionary linguistics}

In our first example, we examine a model from evolutionary linguistics. The model was constructed by Komarova, Niyogi, and Nowak [3] to study the circumstances under which a single dominant language can evolve, given the fact that language learning is error prone. Komarova et al. only employ the birth process, but the model is easily modified to use DP or LD instead.

We will use this model to show that the different updating rules can lead to different topologies of the phase spaces of some models with high mutation rates.

Consider a finite population of agents. Each agent speaks exactly one of $n$ different languages. Define the fitness $f_{i}$ of an individual speaking language $i$ as

$$
f_{i}=f_{0}+\frac{1}{2} \sum_{j=1}^{n} a_{i j} x_{j} .
$$

Here $f_{0}>0$ is the background fitness which does not depend on the agent's language, $x_{j}$ is the proportion of the population speaking language $j$, and $A=a_{i j}$ is a fully symmetric mutual comprehension matrix, i. e.,

$$
a_{i i}=1 \quad a_{i j}=a \quad(i \neq j),
$$

with a constant parameter $a$. In every time step, one individual is replaced by another individual according to the updating rule chosen. Mutation is uniform with probability $\mu>0$, such that the offspring has a probability of $q_{i i}=q=1-\mu$ of speaking the same language as their parent, and a probability of $q_{i j}=$ $\frac{\mu}{n-1}$ for each of the other languages.

\section{Birth process}

The BP is the original updating rule used in Ref. [3]. In BP, the probability of an individual of type $j$ dying is $M^{j}(x)=x_{j}$. The birth probability, including mutation, is $F^{j}(x)=\phi^{-1} \sum_{i} f_{i} x_{i} q_{i j}$, where $\phi=\sum_{m=1}^{n} f_{m} x_{m}$. Thus the BP with mutation corresponds to the replicator-mutator differential equation [36] used by Komarova et al. [3]

$$
\dot{x_{j}}=\sum_{i} f_{i} x_{i} q_{i j}-\phi x_{j}
$$

under a dynamic rescaling of time which does not change the equilibria of the model.

Komarova et al. have proved that for all values of the model parameters $a, \mu, n, f_{0}$, there is an equilibrium in which every language has the same frequency $x_{j}=\frac{1}{n}$. Stable asymmetric equilibria with one dominant language exist if and only if the discriminant

$$
\begin{aligned}
D= & D^{\mathrm{BP}}=4\left[-1-a(n-2)-f_{0}(n-1)\right](1-q)(n-1) \\
& \times(1-a)+(1-a)^{2}[1+(n-2) q]^{2}
\end{aligned}
$$

is positive. For $D>0$ then also an asymmetric third type of equilibrium exists such that one language $i$ has frequency $x_{i}=x_{e q}\left(a, \mu, n, f_{0}\right)<\frac{1}{2}$ and all other languages have identical frequency $\frac{1-x_{i}}{n-1}$. If $x_{i}<\frac{1}{n}$, this asymmetric equilibrium is stable and the symmetric equilibrium is unstable. Otherwise, the symmetric equilibrium is an attractor and the asymmetric equilibrium is a repeller. The equilibrium structures in this model, as a function of the mutation rate $\mu$, are shown in Fig. 1. [3]

We will now generalize the model in order to derive its behavior under the DP and LD updating rules.

\section{Death process}

For the DP, we have $F^{j}(x)=\sum_{i} x_{i} q_{i j}$ and $M^{j}(x)=$ $\psi^{-1} \frac{1}{f_{j}} x_{j}$. Here $\psi=\sum_{m=1}^{n} \frac{x_{m}}{f_{m}}$ is again a normalizing factor. Thus the system of analytical ordinary differential equations corresponding to (14) is given by

$$
\dot{x_{j}}=\sum_{i} x_{i} q_{i j}-\psi^{-1} \frac{1}{f_{j}} x_{j} .
$$

We apply the computations from Ref. [3] to this modified model. An equilibrium is given by $\dot{x}_{j}=0$. Using Eqs. (12), 

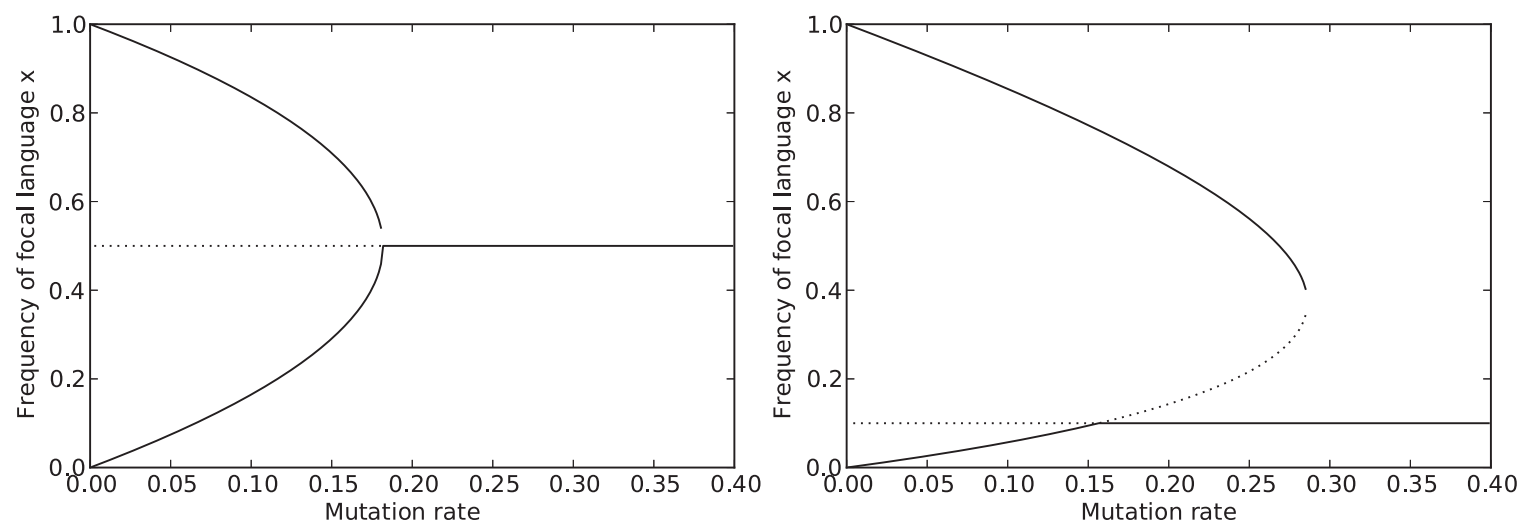

FIG. 1. Equilibria of model 1 with BP as updating rule for varying $\mu$ and $a=0.2, f_{0}=0.1$. The points on the solid line are stable equilibria for those parameter values, the points on the dotted line are unstable equilibria. (a) For $n=2$; (b) for $n=10$.

(13), and (16), this becomes

$$
\frac{1}{(1-a) x_{j}+a+f_{0}} x_{j}=\sum_{m=1}^{n} \frac{x_{m}}{(1-a) x_{m}+a+f_{0}} \sum_{i} x_{i} q_{i j} .
$$

Without loss of generality, let $x_{1}=x$ and $x_{i}=\frac{1-x}{n-1}$ for $i \neq 1$. Then

$$
\begin{aligned}
\frac{1}{(1-a) x+a+f_{0}} x= & \sum_{m=2}^{n} \frac{1-x}{(n-1)\left(a+f_{0}\right)+(1-x)(1-a)} \\
& \times\left(\sum_{i=2}^{n} \frac{1-x}{n-1} \frac{1-q}{n-1}+x q\right),
\end{aligned}
$$

which has the solutions $x=\frac{1}{n}$ and

$$
\begin{aligned}
x= & \frac{ \pm \sqrt{D^{\mathrm{DP}}}+(1-a)(n q-1)}{2(1-a)(n q-1)} \\
D^{\mathrm{DP}}= & -(a-1)(n q-1)\left(3 a n q+4 f_{0} n q-4 a n-4 a q\right. \\
& \left.-4 f_{0} n-4 f_{0} q+n q+5 a+4 f_{0}-1\right) .
\end{aligned}
$$

We have thus obtained a similar condition as in the BP case, stating that asymmetric equilibria with one focal language of frequency $x$ and all other languages having frequency $\frac{1-x}{n-1}$ exist if and only if the discriminant

$$
\begin{aligned}
D^{\mathrm{DP}}= & D^{\mathrm{BP}}+4(q-1)^{2}(n-1)(1-a) \\
& \times\left[\left(a+f_{0}\right) n+(1-a)\right]>0 .
\end{aligned}
$$

\section{Link dynamics}

In the case of the LD update rule, the limit of the stochastic process for increasing population size is given by the ordinary differential equation

$$
\dot{x_{j}}=\sum_{i}\left(\sum_{k} \frac{f_{i}}{f_{i}+f_{k}} x_{k}\right) x_{i} q_{i j}-\left(\sum_{k} \frac{f_{k}}{f_{j}+f_{k}} x_{k}\right) x_{j}
$$

because $\quad M^{j}(x)=\left(\sum_{k} \frac{f_{k}}{f_{j}+f_{k}} x_{k}\right) x_{j} \quad$ and $\quad F^{j}(x)=$ $\sum_{i}\left(\sum_{k} \frac{f_{i}}{f_{i}+f_{k}} x_{k}\right) x_{i} q_{i j}$ for LD.
Using the same argumentation as before, it follows from this differential equation that the equilibria are given by

$$
0=\sum_{k}\left(\sum_{i} \frac{f_{i}}{f_{i}+f_{k}} x_{i} q_{i 1}\right) x_{k}-\left(\sum_{k} \frac{f_{k}}{f_{1}+f_{k}} x\right) x_{k} .
$$

Using Eqs. (12), (13), and (16), this is a cubic equation in $x$,

$$
\begin{aligned}
0= & \frac{1-q}{2}\left[x^{2}-\frac{(1-x)^{2}}{n-1}\right] \\
& +x(1-x)\left\{q\left[(1-a) x+a+f_{0}\right]+\frac{2-n-q}{n-1}\right. \\
& \left.\times\left[(1-a) \frac{1-x}{n-1}+a+f_{0}\right]\right\}
\end{aligned}
$$

with solutions $x=\frac{1}{n}$ and

$$
\begin{aligned}
x= & \frac{(1-a)(n-1) q \pm \sqrt{D^{\mathrm{LD}}}}{(1-a)(n q+n-2)} \\
D^{\mathrm{LD}}= & D^{\mathrm{BP}}+(q-1)^{2}(n-1)(1-a) \\
& \times\left[2\left(a+f_{0}\right) n+3(1-a)\right]
\end{aligned}
$$

if $D^{\mathrm{LD}}>0$.

\section{Comparison}

Comparing $D^{\mathrm{DP}}$ [Eq. (17)] with $D^{\mathrm{BP}}$ [Eq. (15)], we see that due to the positive difference

$$
\begin{aligned}
D^{\mathrm{DP}}-D^{\mathrm{BP}}= & 4(q-1)^{2}(n-1)(1-a) \\
& \times\left[\left(a+f_{0}\right) n+(1-a)\right]>0
\end{aligned}
$$

the asymmetric equilibria exist for a broader range of parameters in the case of DP than in the case of the BP. In particular, the death process permits a stable asymmetric equilibrium with a dominant language for parameter values that only support a symmetric equilibrium in case of BP.

We see that the discriminant $D^{\mathrm{LD}}$ [Eq. (18)] still has an additional positive addend as compared to the birth-death process discriminant $D^{\mathrm{BP}}$. On the other hand, we have $D^{\mathrm{LD}}<$ $D^{\mathrm{DP}}$ if $q<1$. Thus, just as derived in Eq. (12) for the case of two strategies in the absence of mutation, we see that the link 

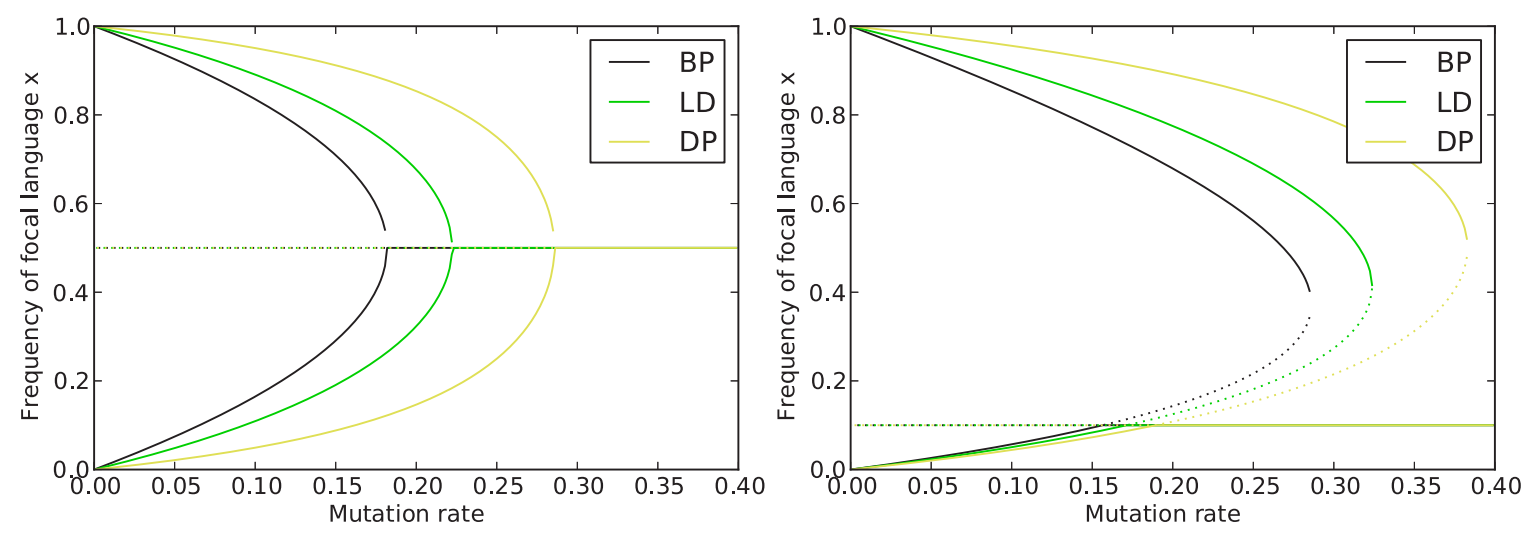

FIG. 2. (Color online) Equilibria of model 1 for varying $\mu$ for $a=0.2, f_{0}=0.1$. The points on the solid line are stable equilibria for those parameter values, and the points on the dotted line are unstable equilibria. (a) For $n=2$; (b) for $n=10$.

dynamics process is a an intermediate updating rule between DP and BP.

In the case of $n=2$ and $\mu>0, D^{\mathrm{DP}}>D^{\mathrm{LD}}>D^{\mathrm{BP}}$ still holds. Thus for a range of parameters, the equilibrium structure under DP differs from the equilibrium structure under BP even for a model with only two languages.

\section{Interpretation}

We conclude that, as shown in Fig. 2, for low mutation rates the behavior of the model under all three updating rules is similar. All updating rules lead to stable asymmetric equilibria at similar values. For larger mutation rates, the quantitative difference between the equilibria under different updating rules increases. When the mutation rate reaches a critical threshold, at $\frac{(1-a)(n-1)}{n\left(a(n-2)+f_{0} n+2\right)} \leqslant \mu<\frac{(1-a)(n-1)}{n\left(a(n-1)+f_{0} n+1\right)}$, the behavior of the model changes qualitatively when changing the updating rule. In this case, both LD and DP permit the existence of asymmetric equilibria, while the BP shows only the stable symmetric equilibrium. For $\frac{(1-a)(n-1)}{n\left(a(n-1)+f_{0} n+1\right)} \leqslant$ $\mu<\frac{2(1-a)(n-1)}{n\left(a(2 n-3)+2 f_{0} n+3\right)}$, the model shows a different qualitative behavior change. For a large area of the parameter space, both BP and LD do not permit the existence of asymmetric equilibria, but the DP still sustains a stable asymmetric equilibrium, in which the dominant language has a frequency of substantially more than half the population. Only for $\mu \geqslant$ $\frac{2(1-a)(n-1)}{n\left(a(2 n-3)+2 f_{0} n+3\right)}$ do all three models show the same behavior again, with only a stable symmetric equilibrium present.

The conclusion from these calculations is that changing the updating rule does not merely perturb the equilibria but also that it can also give rise to different equilibrium structures. Thus we have illustrated that for high mutation rates the number and structure of evolutionary stable states in a model can depend on the choice of updating rule.

\section{Model 2: Abstract evolutionary game theory}

For sufficiently low mutation rates, we have seen that the equilibria of the previous example change only very slightly under different updating rules. The locations and types (attractor, repeller, or saddle) of the equilibria in the absence of mutation are dependent only on the fitness functions of the alleles. It might therefore be a reasonable hypothesis that knowledge of the locations of saddle points and stable and unstable equilibria in the fitness landscape is sufficient to fix the topology of the basins of attraction in this landscape. This is, however, not the case.

We now introduce a different model from the field of abstract evolutionary game theory. We will use it to illustrate that the topology of the basins of attraction of the stable equilibria can change when choosing a different updating rule.

Consider a well-mixed population of size $n$. Each individual in the population has one of the alleles $\mathrm{A}, \mathrm{B}$, or $\mathrm{C}$. The fitness of an individual of type $\mathrm{A}, \mathrm{B}$, and $\mathrm{C}$, respectively, are frequency dependent and given by

$$
\begin{aligned}
& f_{\mathrm{A}}=\exp \left(3-3 n_{\mathrm{A}}+\frac{9 n_{\mathrm{B}}}{2}\right) \\
& f_{\mathrm{B}}=\exp \left(7+66 n_{\mathrm{B}}-81 n_{\mathrm{A}} n_{\mathrm{B}}-64 n_{\mathrm{B}}^{2}\right) \\
& f_{\mathrm{C}}=\exp \left(3 n_{\mathrm{A}}-\frac{9 n_{\mathrm{B}}}{2}\right)
\end{aligned}
$$

where $0 \leqslant n_{\mathrm{A}}, n_{\mathrm{B}} \leqslant 1$ are the relative frequencies of alleles $\mathrm{A}$ and $\mathrm{B}$, respectively.

We can study the system in the limit for $n \rightarrow \infty$, as described in Sec. III A. We thereby obtain ordinary differential equations for the behavior of the system. Numerically solving these ODEs yields the basins of attraction shown in Fig. 4.

Running numerical simulations for this model with $n=50$, starting 500 runs at every location of the simplex and averaging their states when fixated, we obtain the results shown in Fig. 3.

\section{Comparison}

The attractors (filled circles) and repellers (end point of the black trajectory) remain the same under different updating rules. However, while mutation and random drift do not feature in the large mutation limit, the updating rule still has a significant influence on the basins of attraction in the model.

For BP, the majority of states, including the area near the stable mixed equilibrium $M$ between $A$ and $C$ (purple), lie in the basin of attraction of the pure B equilibrium (red). This is apparent both in the numerical and large-population versions of the model. In contrast, given a DP updating rule, the majority of states, including the area around M, lie in the basin of 

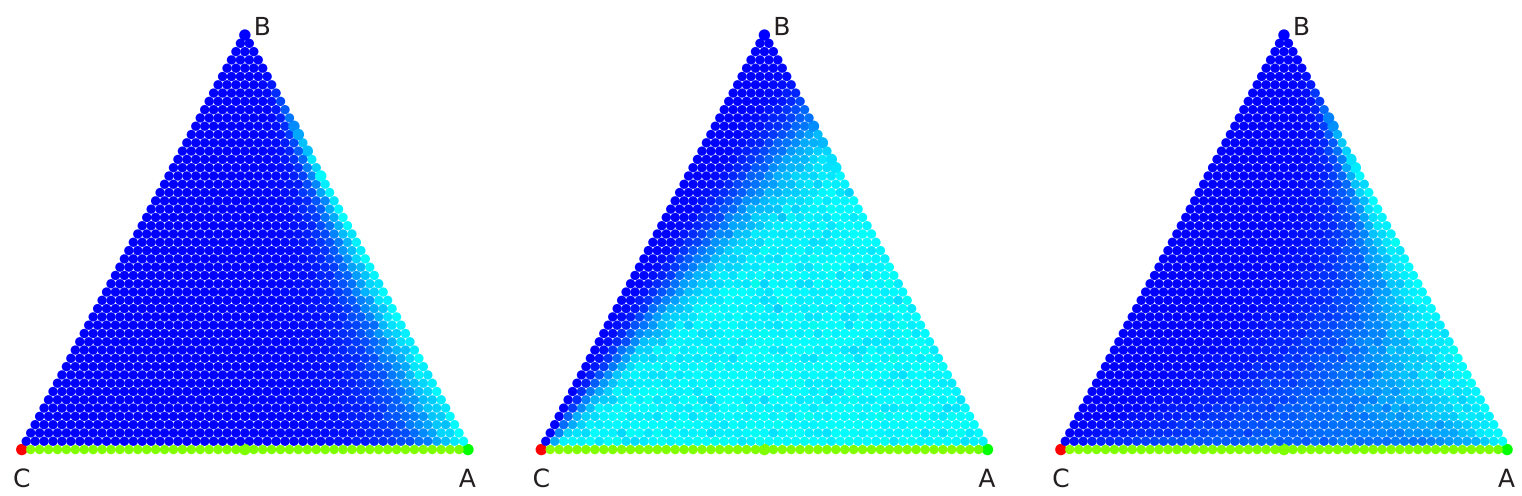

FIG. 3. (Color online) Numerical simulation results of model 2 in barycentric coordinates. In each subfigure, the colors indicate how many runs starting in that location fixate in what state. In all three cases, the only runs fixating in $\mathrm{C}$ (red, bottom left) are those already starting in a fixated C state. All mixtures of of A and C (bottom line) fixate in A (green, bottom right). The rest of the state space is split between states that nearly exclusively fixate in pure B [blue (dark gray)] and states that fixate in either A or B [cyan (bright gray)], with darker colors indicating a higher probability to fix in B. (a) BP; (b) DP; (c) LD.

attraction of the stable mixed equilibrium between $\mathrm{A}$ and $\mathrm{B}$ (green). We also see that for LD, the area near M lies in the basin of attraction of $\mathrm{B}$ but close to the boundary between the basins of attraction.

Therefore, we expect that small mutations away from the mixed stable $\mathrm{A}$ and $\mathrm{C}$ state will lead to fixation in $\mathrm{B}$ for the birth process, and to the stable equilibrium between $\mathrm{A}$ and $\mathrm{B}$ for $\mathrm{DP}$. In the case of $\mathrm{LD}$, the long-term behavior of the system depends significantly on the stochastic motion of the system. These behaviours are indeed seen in the results of the numerical simulations, Fig. 3.

Heuristically, we see that in the BP, trajectories point towards strong attractors, which is most obivous in this example when considering the pure B equilibrium. The DP, on the other hand, is dominated by the repellers, which can be seen from the fact that all trajectories point away from the unstable pure $\mathrm{C}$ equilibrium. Even close to the strong attractor $\mathrm{B}$, the leftmost trajectory follows a nearly straight line away from $\mathrm{C}$. The LD updating rule shows both, with the precise behavior locally depending on the strength of attraction or repulsion. With only two strategies in a system, the geometry is one dimensional, and therefore there is no difference between a focus on attractors or repellers, so this effect can only be seen for three or more strategies.
In order to obtain geometrically different basins of attraction such as those described in this example, the system has to contain at least two attractors. Close to an unstable equilibrium between these two attractors, the fitnesses of the alleles must be of different orders of magnitude. In the geometric interpretation, there is a large angle between vectors describing the trajectory away from a strong repeller and that toward a strong attractor. In the example given above, the relevant unstable mixed equilibrium is $n_{\mathrm{A}} \approx 0.176$, $n_{\mathrm{B}} \approx 0.824, n_{\mathrm{C}}=0$. Away from this in the direction of $\mathrm{C}$, $f_{\mathrm{A}}$ decreases, but $f_{\mathrm{B}}$ increases significantly, while $f_{\mathrm{C}}$ is very low. Thus the boundary of the basin of attraction has a different orientation close to that point and can differ significantly when followed further.

However, different updating rules can still lead to different behavior in a system not containing equilibria of these types, as the following example will illustrate.

\section{Model 3: Public goods game with punishment}

The previous example shows that the impact of the choice of updating rule can be quite large even for stochastic simulations. The shape of the basins of attraction is however strongly dependent on the precise coefficients, and the different
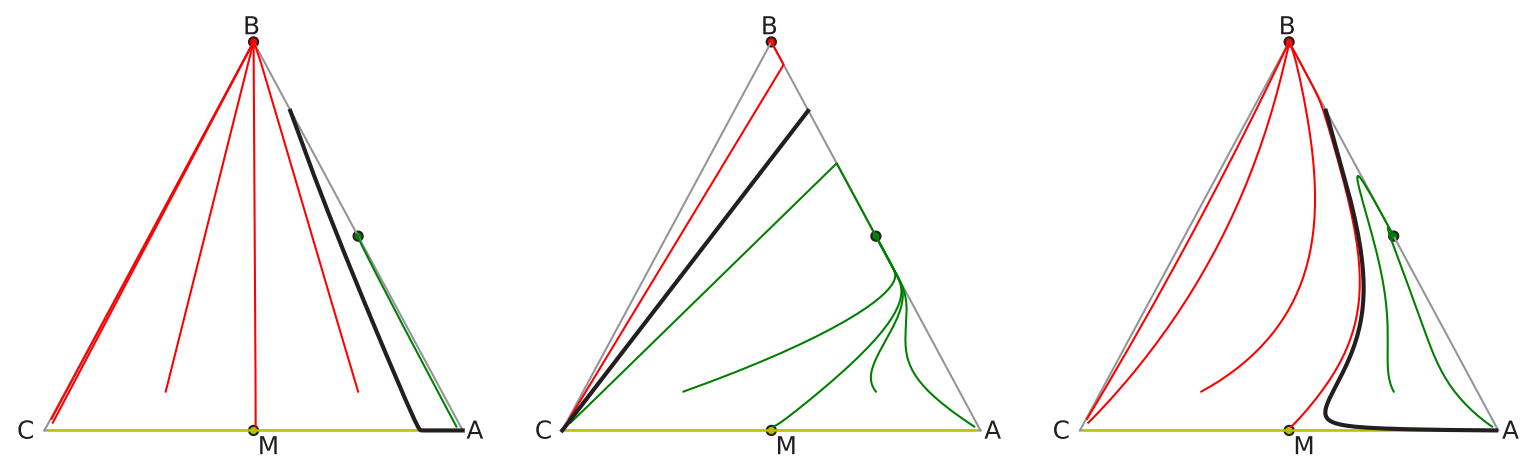

FIG. 4. (Color online) Areas of convergence for BP, DP, and LD in Model 2 in barycentric coordinates. The thick black line denotes the boundary between the basins of attraction. Other lines indicate trajectories through the population state space ending in the mixed $\mathrm{A}$ and $\mathrm{C}$ (yellow, bottom line of the triangle), pure B (red, all trajectories left of the boundary line), and mixed A and B (green, all trajectories right of the boundary line) equilibria. 

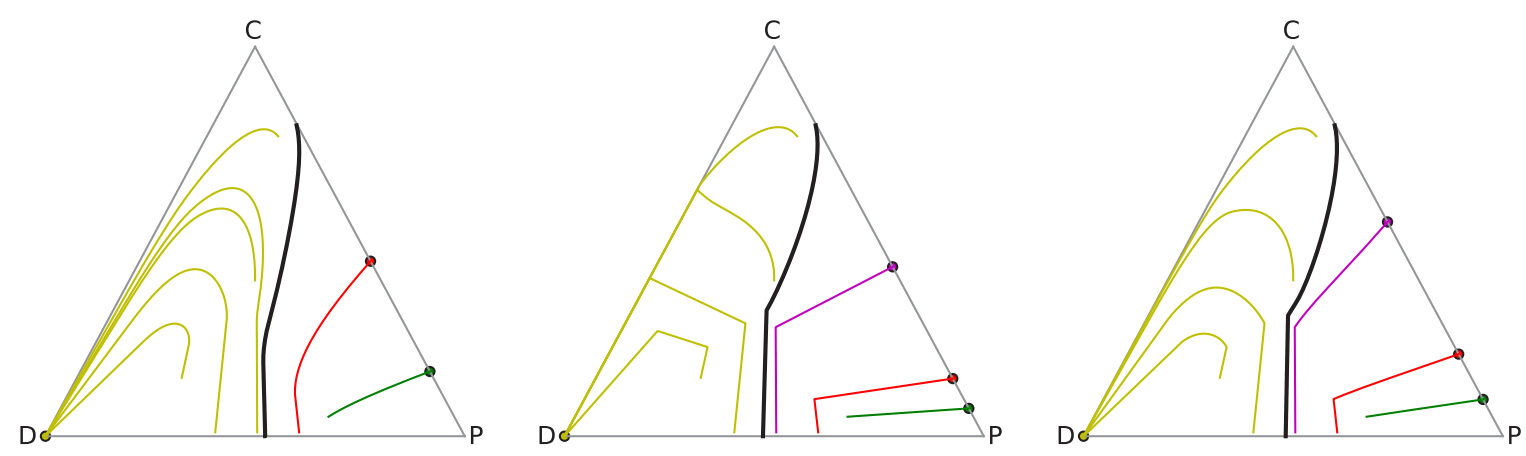

FIG. 5. (Color online) Areas of convergence for BP, DP, and LD in Model 3 in barycentric coordinates for $r=2.8, c=0.8, \beta=1.5$, and $\gamma=0.6$. The thick black line denotes the boundary between the basins of attraction. Other lines indicate trajectories through the population state space ending up in the pure D equilibrium (yellow, all trajectories left of the boundary line) and on the neutral line between C and $\mathrm{P}$ (all trajectories right of the boundary line). Note the trajectory starting near the even mixture of $\mathrm{D}$ and $\mathrm{P}$ ends up in the pure $\mathrm{D}$ equilibrium in the $\mathrm{BP}$, while it ends up on the neutral line for the other updating rules.

behavior occurs largely due to strong selection caused by the exponential function in Eq. (19).

The public goods game is an $N$-player game frequently used in theoretical [37] and experimental economics [38,39] to model the tragedy of the commons. The game is a natural extension of the weak prisoner's dilemma to an arbitrary number of players.

In one PGG round, players can choose to invest in a common good or not. Contributing cooperators (C) pay a fixed cost $c$. All contributions are added up, multiplied by a factor $r>1$, and distributed among all participants, no matter if they were contributing cooperators or noncontributing defectors (D). For investigations into the evolution of cooperation, this simple model is often extended by two additional strategies [40].

Peer punishers $(\mathrm{P})$ choose to punish defectors as well as contribute to the public goods. After resources have been redistributed, they pay a high cost $\gamma$ per defector present in the game. By doing so, they inflict an even higher utility loss, the fine $\beta$, on each defector present in the game. [41,42]

Many workers in population dynamics literature add loners (L) as additional strategy. Players who follow this strategy gain a small frequency-independent payoff $\lambda$. Loners opt out of the game altogether, reflecting the fact that participating in a specific group is also a choice which can be studied using game theoretical methods $[43,44]$.

Further extensions to the basic public goods game have been studied in the literature, such as pool punishers who pay a constant fee for the punishment of all defectors $[42,45]$ or strategies that reward cooperators in addition to or alternatively to punishing defectors $[37,46]$.

If a strategy would have a negative payoff in a given state, we will assume a fitness of 0 . Otherwise, the fitness is taken to be proportional to the payoff. The fitnesses for the four strategies are then given by

$$
\begin{aligned}
& f_{\mathrm{C}}=\max \left(0, r c \frac{n_{\mathrm{C}}+n_{\mathrm{P}}}{n_{\mathrm{C}}+n_{\mathrm{D}}+n_{\mathrm{P}}}-c\right), \\
& f_{\mathrm{D}}=\max \left(0, r c \frac{n_{\mathrm{C}}+n_{\mathrm{P}}}{n_{\mathrm{C}}+n_{\mathrm{D}}+n_{\mathrm{P}}}-n_{\mathrm{P}} \beta\right), \\
& f_{\mathrm{P}}=\max \left(0, r c \frac{n_{\mathrm{C}}+n_{\mathrm{P}}}{n_{\mathrm{C}}+n_{\mathrm{D}}+n_{\mathrm{P}}}-c-n_{\mathrm{D}} \gamma\right), \quad f_{\mathrm{L}}=\lambda .
\end{aligned}
$$

Figure 5 shows the behavior of the mean-field theory for this model in the D-C-P plane. The parameter values are $r=2.8$, $c=0.8, \beta=1.5$, and $\gamma=0.6$. The state where all individuals follow the D strategy is an attractor. In the absence of defectors, cooperators and punishers are indistinguishable. Every state not in the basin of attraction of the pure $\mathrm{D}$ state will converge towards a mixed $\mathrm{C}$ and $\mathrm{P}$ state. However, the fitness functions close to the unstable equilibrium on the D-P line are $f_{\mathrm{C}}>0$, $f_{\mathrm{D}}=0$, and $f_{\mathrm{P}}=0$. It is therefore not surprising that the geometry of the basin of attraction changes only slightly when changing the updating rule.

Changing the update rule used for the public goods game will therefore have an influence on the long-term behavior of the system only when stochasticity plays a significant role. We will now investigate the effect of different updating rules on the stationary distribution of the Markov chain.

Figure 6 shows the stationary distribution of the Markov chain induced by the public goods game with punishment and different updating rules. The values have been numerically obtained through power iteration of the transition probability matrix P. Darker color indicates a higher probability of the corresponding state in the stationary distribution. The locations of the global minima of probability according to BP and DP differ significantly. The stationary distribution obtained from LD shows two local minima roughly corresponding to the global minima of BP and DP, again supporting the hypothesis that LD generally behaves as a mixture of BP and DP. We numerically calculated the average frequencies for $\mathrm{D}, \mathrm{C}$, and $\mathrm{P}$ in the stationary distribution. For the BP, we obtain a defector-dominated population with $n_{\mathrm{D}}=0.748$. The average relative frequencies for the cooperative strategies amount to $n_{\mathrm{C}}=0.0899$ and $n_{\mathrm{P}}=0.0899$. In contrast to the $\mathrm{BP}$, where nearly three-quarters of the population are defectors, less than half of the population follow a noncooperative strategy in the DP, with $n_{\mathrm{D}}=0.428$. The cooperative strategies amount to $n_{\mathrm{P}}=0.406, n_{\mathrm{C}}=0.166$. The LD updating rule is again intermediate between BP and DP, yielding $n_{\mathrm{P}}=0.314, n_{\mathrm{C}}=$ 0.145 , and $n_{\mathrm{D}}=0.541$. This means that the change of updating rule alone can change an evolutionary model from being dominated by defectors to including primarily cooperative strategies. 

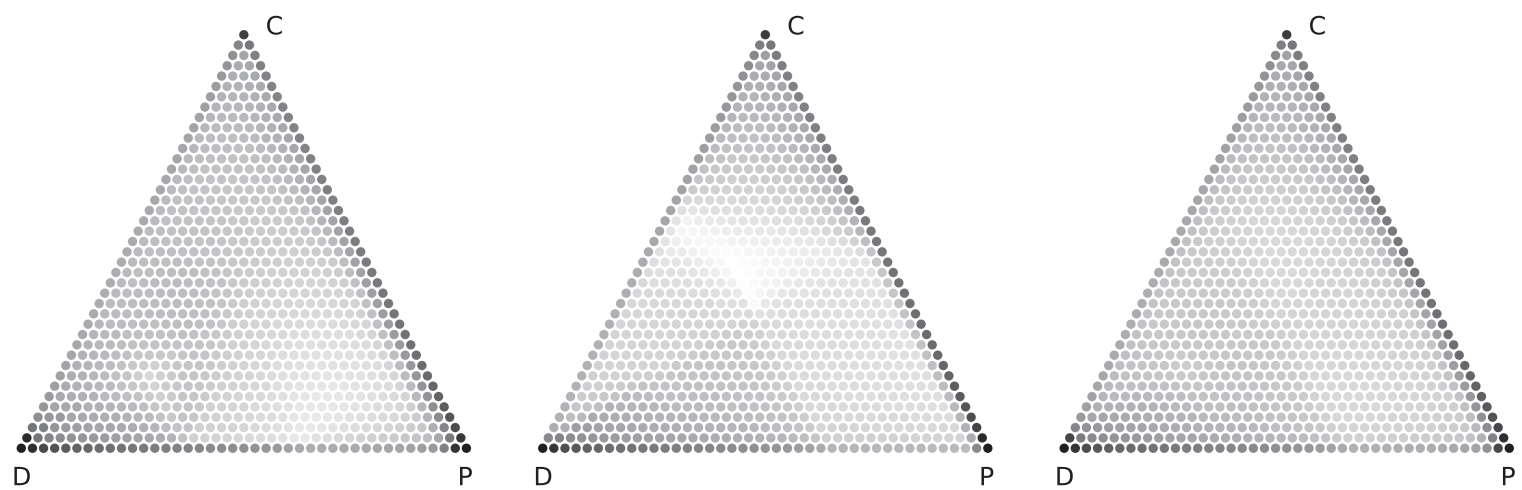

FIG. 6. Stationary distribution of the Markov chain induced by the public goods game with punishment for $N=40, \mu=0.003953$, $r=2.8, c=0.8, \beta=1.5$, and $\gamma=0.6$ and different updating rules. Darker color indicates a higher probability of the corresponding state in the stationary distribution. (a) BP; (b) DP; (c) LD.

\section{DISCUSSION}

In this paper, we have studied the impact of the choice of updating rule, that part of a model which describes the manner in which a population changes from generation to generation, on the results of well-mixed evolutionary models. Our work complements known results for structured populations, which are able to more closely represent many real-world systems. It has previously been established in the literature that in populations with nonhomogenous graph structures, where nodes have different numbers of links leading to them, changing the updating rule can change the dynamics of an evolutionary model [29,30].

Regular graph structures, in particular square or cubic lattices (spatial games, e. g., Cox and Griffeath in Ref. [1]) or "well-mixed" populations (e. g., Komarova et al. in Ref. [3]) have been widely used for models in evolutionary game theory. Both lattices and well-mixed populations are homogenous graphs. Social networks, however, cannot be represented by regular graphs. Rather, they have a degree distribution with a heavy tail, where many nodes have low degrees, but very few nodes have a very high degree each. In the field of evolutionary game theory inhomogenous graphs have been successfully used to study the evolution of cooperation [4,17,47-50], but the impact of changing the updating rule on these results is not fully clear.

Here we have turned our attention back to the most fundamental case of well-mixed populations. We have explored three example models in which the choice of updating rule has a severe impact on the system's behavior, even in the absence of a structured population. They highlight three additional constraints on results suggesting updating rule equivalence. We have found that for high mutation rates, different updating rules can lead to the presence of nontrivially different equilibria in the system. Even in the absence of mutation, we find that when a system has multiple stable equilibria, their basins of attraction can topologically differ under different updating rules. Differing behavior can be seen in the mean-field approximation as well as in the stochastic theory. We have further shown that the choice of updating rule can significantly influence relative frequencies of the strategies in the long run if more than two strategies interact nontrivially.

We are therefore led to conclude that the equivalence of updating rules should not be assumed in general. Any generalization away from investigating fixation probabilities of two-strategy games on homogenous graphs with low mutation may lead to model results that depend on the updating rule.

The properties of evolutionary models with different updating rules outlined in this article have a concrete impact on modeling real-world evolutionary processes. It falls to researchers to choose the updating rule based on qualities of the system to be explored. For example, one interpretation sees the three rules as representing different population dynamics. If we consider the death of a random individual, uniformly chosen, to be a normalization term to keep the population size constant, it is obvious that the dynamics obtained from a BP model approximate a growing population. By the same argument, the DP updating rule, on the other hand, can be considered a renormalized approximation to a shrinking population and may be significantly better suited for systems where detrimental mutations go extinct quickly. The link dynamics updating rule generally behaves like a mean between the BP and DP and appears particular suited for models where direct competition between individuals plays a significant role for the dynamics of the system.

It is well known that selection in growing and shrinking populations are connected to different selection mechanisms [51,52]. When modeling concrete evolutionary processes, it is therefore important to choose the updating rule based on the population dynamics and selection mechanisms of the reference system.

The study of updating rules in the context of evolutionary game theory is thus important in order to build more realistic models of the systems studied. Far more is known about selection on birth both in models and in reality. Birth processes are more widespread in modeling, and birth and progeny are easier to study than natural deaths. This applies even more so to cultural variants. Circumstances of their emergence are far more easily observed than those of their demise. Furthermore, in the ecological context of life-history theory it is clear that relative birth rate and survival probability may be determined by different factors, and models accurately representing this phenomenon may be preferred. Incorporating selection terms on birth and death may lead to coexistence of organisms selected for birth and those selected for survival [53,54]. Understanding the evolutionary dynamics of such systems 
requires a profound understanding of the impact of selection on birth versus selection of death.

In this article, we have studied only well-mixed populations. However, because it is known that population structure by itself can heavily modify model behavior, and because many real-world systems show significantly nontrivial population structures, future work needs to study the dynamics of structured populations with different updating rules. Models also show the impact of more than two alleles and high mutation rates on structured population models.

In conclusion, our work has highlighted the dangers of blindly following traditional algorithms when designing evolutionary models. Any model should be constructed to best represent the system it explores. In cases where a statement on the updating rule is not implied by a problem it is important to confirm that the updating rule does not have a meaningful impact on the model, while recalling that results for the stochastic and deterministic cases can differ. We refer back to our introduction, in which we noted that not only our understanding of the world but also policies affecting our interactions with it are sometimes informed by evolutionary models. It would be unfortunate to find ourselves misled by unexpected quirks of model behavior.

\section{ACKNOWLEDGMENTS}

We thank the EPSRC for Ph.D. funding.
[1] J. T. Cox and D. Griffeath, Ann. Probab. 14, 347 (1986).

[2] D. L. Schwartz, Ann. Probab. 5, 522 (1977).

[3] N. L. Komarova, P. Niyogi, and M. A. Nowak, J. Theor. Biol. 209, 43 (2001).

[4] H. Ohtsuki, C. Hauert, E. Lieberman, and M. A. Nowak, Nature 441, 502 (2006).

[5] J. C. van den Bergh, J. Evol. Econ. 17, 521 (2007).

[6] D. M. Frank and S. Sarkar, PLoS ONE 5, e10688 (2010).

[7] A. Szolnoki and M. Perc, Sci. Rep. 4 (2014).

[8] A. Szolnoki and M. Perc, Phys. Rev. E 89, 022804 (2014).

[9] P. A. P. Moran, Math. Proc. Cambridge 54, 60 (1958).

[10] C. Hauert, J. Theor. Biol. 240, 627 (2006).

[11] P. Clifford and A. Sudbury, Biometrika 60, 581 (1973).

[12] S. Wright, Genetics 16, 97 (1931).

[13] T. Williams and R. Bjerknes, Adv. Appl. Probab. 3, 210 (1971).

[14] M. Bramson and D. Griffeath, Ann. Probab. 9, 173 (1981).

[15] R. A. Holley, T. M. Liggett, et al., Ann. Probab. 3, 643 (1975).

[16] L. E. Blume, Game Econ. Behav. 5, 387 (1993).

[17] J. Poncela, J. Gómez-Gardeñes, L. Floría, and Y. Moreno, New J. Phys. 9, 184 (2007).

[18] M. Newman, Networks: An Introduction (Oxford University Press, Oxford, 2010).

[19] E. Lieberman, C. Hauert, and M. A. Nowak, Nature 433, 312 (2005).

[20] M. Broom and J. Rycht, Proc. R. Soc. A 464, 2609 (2008).

[21] M. Broom, C. Hadjichrysanthou, and J. Rycht, Proc. R. Soc. A 466, 1327 (2010).

[22] S. Tan, J. Lu, and G. Setti, in IECON 2011-37th Annual Conference on IEEE Industrial Electronics Society (IEEE, New York, 2011). pp. 4024-4028.

[23] M. Perc, J. Gómez-Gardeñes, A. Szolnoki, L. M. Floría, and Y. Moreno, J. R. Soc. Interface 10, 80 (2013).

[24] G. Szabó and C. Tôke, Phys. Rev. E 58, 69 (1998).

[25] A. Traulsen, M. A. Nowak, and J. M. Pacheco, Phys. Rev. E 74, 011909 (2006)

[26] B. Wu, C. S. Gokhale, L. Wang, and A. Traulsen, J. Math. Biol. 64, 803 (2012).

[27] L. A. Imhof, D. Fudenberg, and M. A. Nowak, Proc. Natl. Acad. Sci. USA 102, 10797 (2005).
[28] C. E. Tarnita, H. Ohtsuki, T. Antal, F. Fu, and M. A. Nowak, J. Theor. Biol. 259, 570 (2009).

[29] T. Antal, S. Redner, and V. Sood, Phys. Rev. Lett. 96, 188104 (2006).

[30] C. Hadjichrysanthou, M. Broom, and J. Rychtár̆, Dynam. Games Appl. 1, 386 (2011).

[31] H. Ohtsuki and M. A. Nowak, Proc. R. Soc. B 273, 2249 (2006).

[32] T. Antal and I. Scheuring, B. Math. Biol. 68, 1923 (2006).

[33] P. M. Altrock and A. Traulsen, New J. Phys. 11, 013012 (2009).

[34] A. Traulsen, J. C. Claussen, and C. Hauert, Phys. Rev. Lett. 95, 238701 (2005).

[35] H. M. Taylor and S. Karlin, An Introduction to Stochastic Modeling (Academic Press, New York, 1994).

[36] M. A. Nowak and K. Sigmund, Science 303, 793 (2004).

[37] A. Szolnoki and M. Perc, Europhys. Lett. 92, 38003 (2010).

[38] A. E. Roth and J. H. Kagel, The Handbook of Experimental Economics, Vol. 1 (Princeton University Press, Princeton, NJ, 1995).

[39] E. Fehr and S. Gächter, Nature 415, 137 (2002).

[40] J. García and A. Traulsen, PLoS ONE 7, e35287 (2012).

[41] H. Brandt, C. Hauert, and K. Sigmund, Proc. R. Soc. B 270, 1099 (2003).

[42] A. Szolnoki, G. Szabó, and L. Czakó, Phys. Rev. E 84, 046106 (2011).

[43] C. Hauert, S. De Monte, J. Hofbauer, and K. Sigmund, J. Theor. Biol. 218, 187 (2002).

[44] C. Hauert, S. De Monte, J. Hofbauer, and K. Sigmund, Science 296, 1129 (2002).

[45] A. Szolnoki, G. Szabó, and M. Perc, Phys. Rev. E 83, 036101 (2011).

[46] A. Szolnoki and M. Perc, Phys. Rev. X 3, 041021 (2013).

[47] G. Szabó and G. Fath, Phys. Rep. 446, 97 (2007).

[48] M. Perc and A. Szolnoki, BioSystems 99, 109 (2010).

[49] F. C. Santos and J. M. Pacheco, Phys. Rev. Lett. 95, 098104 (2005).

[50] H. Ohtsuki and M. A. Nowak, J. Theor. Biol. 243, 86 (2006).

[51] S. S. Jakob, C. Heibl, D. Roedder, and F. R. Blattner, Mol. Ecol. 19, 1423 (2010).

[52] S. P. Otto and M. C. Whitlock, Genetics 146, 723 (1997).

[53] S. Gubbins and C. A. Gilligan, Proc. R. Soc. B 266, 2539 (1999).

[54] W. J. Bond and J. J. Midgley, Oikos 73, 79 (1995). 